\title{
Spin relaxation due to random Rashba spin-orbit coupling in GaAs (110) quantum wells
}

\author{
Y. Zhou and M. W. Wv \\ Hefei National Laboratory for Physical Sciences at Microscale and Department of Physics, \\ University of Science and Technology of China, Hefei, Anhui, 230026, China
}

(Dated: July 6, 2018)

\begin{abstract}
We investigate the spin relaxation due to the random Rashba spin-orbit coupling in symmetric GaAs (110) quantum wells from the fully microscopic kinetic spin Bloch equation approach. All relevant scatterings, such as the electron-impurity, electron-longitudinal-optical-phonon, electronacoustic-phonon, as well as electron-electron Coulomb scatterings are explicitly included. It is shown that our calculation reproduces the experimental data by Müller et al. [Phys. Rev. Lett. 101, 206601 (2008)] for a reasonable choice of parameter values. We also predict that the temperature dependence of spin relaxation time presents a peak in the case with low impurity density, which originates from the electron-electron Coulomb scattering.

PACS numbers: 72.25.Rb, 71.10.-w, 71.70.Ej, 73.21.Fg
\end{abstract}

Semiconductor spintronics has been an active field of research lately due to the potential application of spinbased devices $\frac{1-3}{-3}$ Recent experiments show that the spin relaxation time (SRT) in (110)-oriented GaAs quantum wells (QWs) is extremely long, and thus the spin dynamics in this system has attracted much attention both experimentally and theoretically. $\underline{-18}$ The physics underlying this effect is the absence of the D'yakonov-Perel' (DP) mechanism, $\frac{19}{,}$ which is the leading spin relaxation mechanism in $n$-type zinc-blende semiconductors. The DP mechanism is from the joint effects of the momentum scattering and the momentum-dependent effective magnetic field (inhomogenous broadening ${ }^{20}$ ) induced by the Dresselhaus ${ }^{21}$ and the Rashba ${ }^{22}$ spin-orbit coupling (SOC). In symmetric GaAs (110) QWs with only the lowest subband occupied, the in-plane component of the spin-orbit field vanishes $\underline{\underline{23}}$ Therefore the DP mechanism cannot affect electrons with spin polarization along the growth direction and the SRT in this system is considerably larger than that in (100) QWs. In most of the previous works, the main reason limiting the SRT is attributed to the Bir-Aronov-Pikus mechanism, 24 which is from the exchange interaction between the electrons and the photo-generated holes. One of the exceptions is the spin noise spectroscopy measurement by Müller et al.,$\frac{15}{,}$ where the excitation of semiconductor is negligible and hence the Bir-Aronov-Pikus mechanism is avoided. They reported the longest SRT in this system which is about 24 ns. Since the DP and Bir-Aronov-Pikus mechanisms are both absent, and the virtual intersubband spin-flip SOC induced spin relaxation is also ruled out due to the relatively high mobility of the samples,$\underline{16}$ the possible reason limiting the SRT is the DP mechanism due to the random Rashba SOC caused by the fluctuations of the donor density, $\underline{25,26}$

As shown by Sherman et al., $, 25,26$ even in symmetric QWs, the unavoidable fluctuations of the concentration of the dopant ions still lead to a random electric field along the growth direction, and hence a random Rashba
SOC at each point of a QW. This random SOC provides an inhomogeneous broadening and induces the DP spin relaxation. The previous investigations ${ }^{25.26}$ on the spin relaxation due to this mechanism are based on the singleparticle theory, and thus the electron-electron Coulomb scattering, which has been shown to be very important for spin relaxation in two-dimensional system, $, 20,27-33$ is missing. In this work, we apply the fully microscopic kinetic spin Bloch equation (KSBE) approach ${ }^{20,28}$ to investigate the spin relaxation due to the random Rashba SOC in symmetric GaAs (110) QWs. Here all relevant scatterings, especially the electron-electron Coulomb scattering, are explicitly included. We will show that our calculation is in good agreement with the experimental data by Müller et al..$\frac{15}{}$ We also predict that the temperature dependence of the SRT presents a peak due to the electronelectron Coulomb scattering in the low-impurity-density case.

We start our investigation from an $n$-type symmetrically modulation-doped GaAs (110) QW with the growth direction along the $z$ axis. The in-plane coordinate axes are set as $x \|[1 \overline{1} 0]$ and $y \|[00 \overline{1}]$. A magnetic field $B$ is applied along the $x$-axis. The well width is small enough so that only the lowest subband is occupied for the temperature and electron density we discuss. The envelope function of the relevant subband is calculated under the finite-well-depth assumption. 28 The barrier layer is chosen to be $\mathrm{Al}_{0.39} \mathrm{Ga}_{0.61} \mathrm{As}$ as the experiment ${ }^{15}$ where the barrier height is $319 \mathrm{meV} \stackrel{\underline{34}}{\text { By }}$ using the nonequilibrium Green's function method, $\stackrel{35}{5}$ the KSBEs can be constructed as: 20

$$
\partial_{t} \hat{\rho}_{\mathbf{k}}=\left.\partial_{t} \hat{\rho}_{\mathbf{k}}\right|_{\text {coh }}+\left.\partial_{t} \hat{\rho}_{\mathbf{k}}\right|_{\text {scat }},
$$

where $\hat{\rho}_{\mathbf{k}}$ represents the electron single-particle density matrix, whose diagonal and off-diagonal elements describe the electron distribution function $f_{\mathbf{k} \sigma}$ and spin coherence $\rho_{\mathbf{k}}$ respectively. The scattering term $\left.\partial_{t} \hat{\rho}_{\mathbf{k}}\right|_{\text {coh }}$ consists of the electron-impurity, electron-longitudinaloptical (LO)-phonon, electron-acoustic (AC)-phonon 
and electron-electron Coulomb scatterings, whose expressions are given in detail in Ref. 28. The coherent term can be written as ( $\hbar \equiv 1$ throughout this paper)

$$
\left.\partial_{t} \hat{\rho}_{\mathbf{k}}\right|_{\mathrm{coh}}=-i\left[\mathbf{h}(\mathbf{k}) \cdot \hat{\boldsymbol{\sigma}}+\hat{\Sigma}_{\mathrm{HF}}(\mathbf{k}), \quad \hat{\rho}_{\mathbf{k}}\right],
$$

in which $[A, B] \equiv A B-B A$ is the commutator. $\hat{\Sigma}_{\mathrm{HF}}(\mathbf{k})$ is the effective magnetic field from the Coulomb HartreeFock contribution ${ }^{20} \mathbf{h}(\mathbf{k})$ represents the spin-orbit field composed of the Dresselhaus 21 and Rashba 22 terms. The Dresselhaus spin-orbit field in (110) QWs reads

$$
\mathbf{h}_{\mathrm{D}}(\mathbf{k})=\gamma_{D}\left(0,0, \frac{k_{x}}{2}\left(k_{x}^{2}-2 k_{y}^{2}-\left\langle k_{z}^{2}\right\rangle\right)\right) .
$$

Here $\gamma_{\mathrm{D}}$ denotes the Dresselhaus SOC coefficient and $\left\langle k_{z}^{2}\right\rangle$ stands for the average of the operator $-(\partial / \partial z)^{2}$ over the electronic state of the lowest subband. The effective magnetic field from the Rashba SOC can be written as

$$
\mathbf{h}_{\mathrm{R}}(\mathbf{k})=\alpha_{\mathrm{R}}\left(k_{y},-k_{x}, 0\right)
$$

with $\alpha_{\mathrm{R}}$ representing the Rashba SOC coefficient. To incorporate the effect of the random Rashba SOC, we assume that $\alpha_{\mathrm{R}}$ satisfies the Gaussian distribution function $P\left(\alpha_{\mathrm{R}}\right)=\frac{1}{\sqrt{2 \pi} \Delta_{\mathrm{R}}} e^{-\alpha_{\mathrm{R}}^{2} / 2 \Delta_{\mathrm{R}}^{2}}$ with $\Delta_{\mathrm{R}}$ being the standard deviation of the distribution function $\frac{36}{\underline{6}}$ We divide the regime of $\alpha_{\mathrm{R}}$ from $-\alpha_{\text {cut }}$ to $\alpha_{\text {cut }}$ equidistantly by $N_{\mathrm{R}}$ odd nodes, where $\alpha_{\text {cut }}$ is the cutoff value of $\alpha_{R}$ regime. For each node of $\alpha_{\mathrm{R}}$, we obtain the temporal evolutions of the electron distribution function by numerically solving the KBSEs. The SRT is obtained by the slope of the envelope of the coherently summed spin polarization

$$
S_{z}=\sum_{\alpha_{\mathrm{R}}} \Delta \alpha_{\mathrm{R}} P\left(\alpha_{\mathrm{R}}\right) \sum_{\mathbf{k}} \frac{1}{2}\left[f_{\mathbf{k} \uparrow}\left(\alpha_{\mathrm{R}}\right)-f_{\mathbf{k} \downarrow}\left(\alpha_{\mathrm{R}}\right)\right]
$$

with $\Delta \alpha_{\mathrm{R}}=2 \alpha_{\text {cut }} /\left(N_{\mathrm{R}}-1\right)$. It is checked that $\alpha_{\text {cut }}=$ $1.6 \mathrm{meV} \cdot \AA$ and $N_{\mathrm{R}}=15$ is sufficient for the convergence of our calculation.

First, we compare the calculation via the KSBE approach with the experimental data in Ref. 15. In Fig. 1, we plot the SRT calculated from the KSBEs together with the experimental data as function of the applied magnetic field. Here temperature $T=20 \mathrm{~K}$, well width $a=16.8 \mathrm{~nm}$ and electron density $N_{e}=1.8 \times 10^{11} \mathrm{~cm}^{-2}$ as indicated in the experiment. 15 Impurity density $N_{i}=$ $0.01 N_{e}$ is obtained by fitting the transport mobility given in Ref. 15. It is shown that our calculation agrees well with the experimental data for various magnetic field strength. The fitting gives $\Delta_{\mathrm{R}}=0.195 \mathrm{meV} \cdot \AA$ and

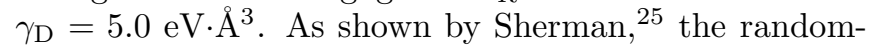
ness of the Rashba SOC is related to the effective distance between the QW conducting sheet and the dopant layer $z_{0}$. The relationship can be written as

$$
\Delta_{\mathrm{R}}^{2}=\left\langle\alpha^{2}\right\rangle=\zeta^{2}\left(\frac{e}{\epsilon}\right)^{2} \frac{\pi N_{e}}{2 z_{0}^{2}}
$$

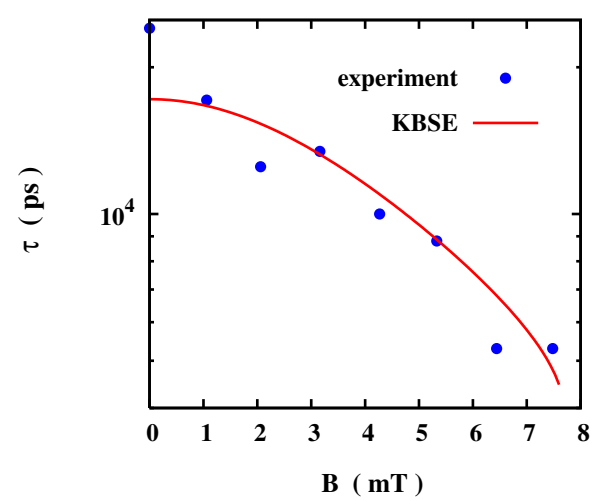

FIG. 1: (Color online) SRTs from the experimental data in Ref. $15(\bullet)$ and from the KSBEs (solid curve) vs. the applied magnetic field. Here $T=20 \mathrm{~K}, a=16.8 \mathrm{~nm}, N_{e}=1.8 \times$ $10^{11} \mathrm{~cm}^{-2}$ and $N_{i}=0.01 N_{e}$.

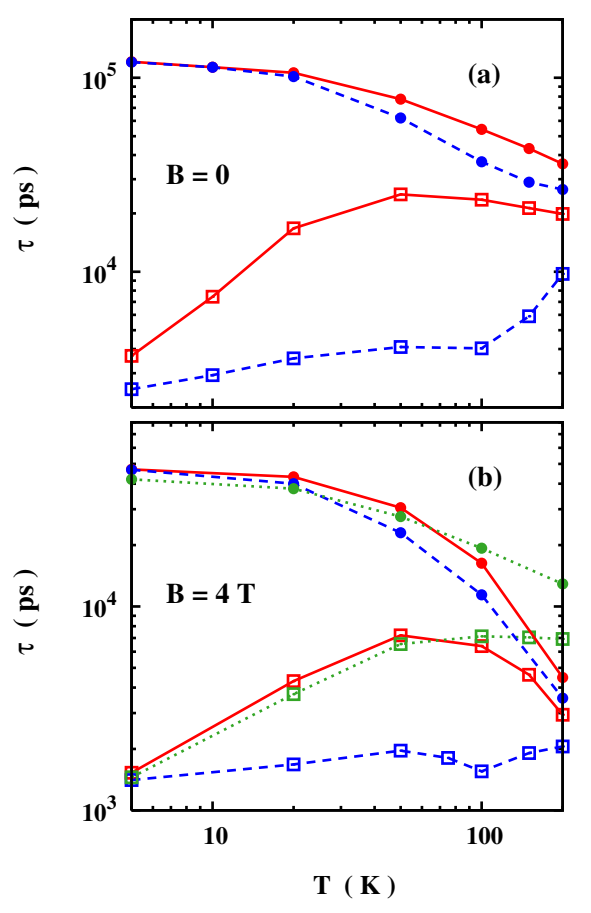

FIG. 2: (Color online) SRTs due to the DP mechanism induced by the random Rashba field vs. temperature $T$ for $B=0$ (a) and $4 \mathrm{~T}$ (b) with impurity densities $N_{i}=N_{e}(\bullet)$ and $0.01 N_{e}(\square)$. Here $a=16.8 \mathrm{~nm}$ and $N_{e}=1.8 \times 10^{11} \mathrm{~cm}^{-2}$. The corresponding Fermi temperature is $75 \mathrm{~K}$. The red solid curves represent the results calculated with all relevant terms; the blue dashed curves are those without the electron-electron Coulomb scattering; the green dotted curves are those without the cubic Dresselhaus SOC. 
where $\zeta=7$ e. $\AA^{2}$ for GaAs $\frac{12}{2}$ From the fitting value of $\Delta_{\mathrm{R}}$, the corresponding effective distance $z_{0}=21 \mathrm{~nm}$, which is consistent with the sample parameter given in Ref. 15. $\frac{37}{3}$ Our best fitted value of $\gamma_{\mathrm{D}}=5.0 \mathrm{eV} \cdot \AA^{3}$ is fairly close to the previous fitting value in GaAs (100) QWs $\left(5.7 \mathrm{eV} \cdot \AA^{3}\right.$ in Ref. $28^{38}$ and $8.6 \mathrm{eV} \cdot \AA^{3}$ in Ref. 29$)$ and the value reported in ab initio calculations (6.4, $\left.8.5 \mathrm{eV} \cdot \AA^{3}\right) \stackrel{39}{3}$ In fact, the measured values of $\gamma_{\mathrm{D}}$ vary from 5.7 to $34.5 \mathrm{eV} \cdot \AA^{3} \underline{28.40}$ This is because the Dresselhaus SOC coefficient is affected by the interface inversion asymmetry: $\stackrel{\underline{41}}{=}$

From Fig. 1, it is also seen that the SRT decreases with the magnetic field. The underlying physics is as following. As first shown by Wu and Kuwata-Gonokami: ${ }^{6}$ in the presence of an in-plane magnetic field, the in-plane and out-of-plane spins are mixed and the Dresselhaus spin-orbit field provides an inhomogeneous broadening which leads to the spin relaxation and dephasing. Therefore the SRT decreases with $B$. For the case with small magnetic field satisfying $\omega_{L} \ll\left(\gamma_{\|}-\gamma_{\perp}\right) / 2, \stackrel{42}{,}$ the effective spin relaxation rate can be written as $\frac{8}{8}$

$$
\gamma_{\mathrm{eff}}=\frac{\gamma_{\|}+\gamma_{\perp}}{2}-\frac{\gamma_{\|}-\gamma_{\perp}}{2} \sqrt{1-\frac{4 \omega_{L}^{2}}{\left(\gamma_{\|}-\gamma_{\perp}\right)^{2}}},
$$

where $\gamma_{\perp}=\Gamma_{\tilde{z} \tilde{z}}$ and $\gamma_{\|}=\Gamma_{\tilde{y} \tilde{y}}$ with $\tilde{z}$ and $\tilde{y}$ being the principle axes of the spin-relaxation-rate tensor $\Gamma$. This definition is due to the fact that the $z$-axis and $y$-axis are not the principle axes of $\Gamma$, when the Rashba and Dresselhaus SOCs are both present $\frac{17,18}{1 t}$ is noted that $\gamma_{\perp}$ differs from $\Gamma_{z z}$ which is the spin relaxation rate neglecting the Dresselhaus SOC. The physics of this effect is similar to that of the influence of the applied magnetic field: $\underline{6}$ the Rashba SOC acts as an in-plane magnetic field, and mixes the in-plane and out-of-plane spins, thus the Dresselhaus SOC can affect the out-of-plane spin relaxation. Our calculation gives the ratio $\gamma_{\|} / \gamma_{\perp}=7.5$, which is consistent with the huge spin dephasing anisotropy observed in experiments $, 8,9,15$

In Fig. 2, we plot the SRT due to the DP mechanism induced by the random Rashba field as function of temperature for $B=0$ (a) and $4 \mathrm{~T}$ (b) with impurity densities $N_{i}=N_{e}$ and $0.01 N_{e}$. The other sample parameters as well as $\Delta_{\mathrm{R}}$ are chosen to be the same as the previous case.$\underline{43}$ We first concentrate on the case without magnetic field [Fig. 2(a)]. It is interesting to see that the SRT exhibits a peak around the Fermi temperature $T_{\mathrm{F}}=E_{\mathrm{F}} / k_{\mathrm{B}}$ in the low-impurity-density case. This peak originates from the electron-electron Coulomb scattering which dominates the momentum scattering. The Coulomb scattering increases with increasing $T$ when $T<T_{F}$ and decreases with $T$ when $T>T_{F}, \stackrel{28,30,44}{,}$ thus the peak appears around the Fermi temperature. It is also seen that the SRT increases with increasing $T$ without the Coulomb scattering. This is because the electron-AC-phonon and electron-LO-phonon scatterings both increase with temperature monotonically. From Fig. 2(a), it is also found that the SRT decreases with increasing $T$ monotonically in the high-impuritydensity case. This is because the electron-impurity scattering dominates the momentum scattering in this case. Since the impurity scattering depends on temperature weakly, the temperature dependence of SRT is mainly determined by the inhomogeneous broadening from the SOC. With an increase of temperature, electrons are distributed at higher momentum states. This leads to the increase of the inhomogeneous broadening and thus a decrease of the SRT.

Then we turn to the case with $B=4 \mathrm{~T}$ [Fig. 2(b)]. For large magnetic field, the SRT is determined by $\gamma_{\text {eff }}=\frac{\gamma_{\|}+\gamma_{\perp}}{2}$. In consistence with the anisotropy factor $\gamma_{\|} / \gamma_{\perp}=7.5$, it is seen that the SRT in this case is three to four times smaller than that in the case without magnetic field. The temperature dependence is similar to the case with $B=0$ : the SRT presents a peak around $T_{F}$ in the low-impurity-density case, while decreases with increasing $T$ monotonically in the high-impurity-density case. The only difference is that the SRT decreases faster at high temperature in the case with high magnetic field. This is because the contribution to the inhomogeneous broadening from the cubic Dresselhaus SOC becomes significant for large well width and high temperature. ${ }^{27} \mathrm{By}$ comparing the SRT without the cubic Dresselhaus SOC, it is found that the effect of the cubic term increases with $T$ faster than the linear Dresselhaus term and the Rashba term (both are linear). This accelerates the decrease of the SRT at high temperature. It is also seen that the SRT without the Coulomb scattering for $N_{i}=0$ first increases then decreases and again increases with increasing $T$. This effect is due to the competition of the increase in the inhomogeneous broadening and the increase in the scattering. When $T<50 \mathrm{~K}$, the contribution from the linear SOC term is dominant and hence the increase of the inhomogeneous broadening with rising temperature is slower than that of the electron-ACphonon and electron-LO-phonon scatterings. This leads to the increase in the SRT. For temperature between $50 \mathrm{~K}$ to $200 \mathrm{~K}$, the contribution of the cubic SOC term is comparable to that from the linear ones. Thus the effect of the inhomogeneous broadening increases with increasing $T$ faster than the electron-AC-phonon scattering but slower than the electron-LO-phonon scattering. By further noticing that the electron-LO-phonon scattering surpasses the electron-AC-phonon scattering when $T>100 \mathrm{~K}$ [see the dashed curve with square in Fig. 2(a)], one can understand that the SRT decreases with increasing $T$ when $50 \mathrm{~K}<T<100 \mathrm{~K}$ but increases when $T>100 \mathrm{~K}$.

In conclusion, we have investigated the SRT due to the DP mechanism induced by the random Rashba field in symmetric GaAs (110) QWs via the fully microscopic KBSE approach, where all the relevant scatterings, especially the electron-electron Coulomb scattering, are explicitly included. We show that our calculation is in good agreement with the experimental data. We also find that the Coulomb scattering makes marked contribution to 
the spin relaxation. It is predicted that the temperature dependence of the SRT exhibits a peak in the lowimpurity-density case, regardless of the applied magnetic field. This peak is from the nonmonotonic temperature dependence of the electron-electron Coulomb scattering.
This work was supported by the National Natural Science Foundation of China under Grant No. 10725417, the National Basic Research Program of China under Grant No. 2006CB922005 and the Knowledge Innovation Project of Chinese Academy of Sciences.
* Author to whom correspondence should be addressed; Electronic address: mwwu@ustc.edu.cn.

${ }^{1}$ F. Meier and B. P. Zakharchenya, Optical Orientation (North-Holland, Amsterdam, 1984).

2 S. A. Wolf, D. D. Awschalom, R. A. Buhrman, J. M. Daughton, S. von Molnár, M. L. Roukes, A. Y. Chtchelkanova, and D. M. Treger, Science 294, 1488 (2001).

3 Semiconductor Spintronics and Quantum Computation, edited by D. D. Awschalom, D. Loss, and N. Samarth (Springer-Verlag, Berlin, 2002); I. Žutić, J. Fabian, and S. Das Sarma, Rev. Mod. Phys. 76, 323 (2004); J. Fabian, A. Matos-Abiague, C. Ertler, P. Stano, and I. Žutić, Acta Phys. Slov. 57, 565 (2007); Spin Physics in Semiconductors, edited by M. I. D'yakonov (Springer, Berlin, 2008); and references therein.

${ }^{4}$ Y. Ohno, R. Terauchi, T. Adachi, F. Matsukura, and H. Ohno, Phys. Rev. Lett. 83, 4196 (1999); Physica E 6, 817 (2000).

5 T. Adachi, Y. Ohno, F. Matsukura, and H. Ohno, Physica E 10, 36 (2001).

${ }^{6}$ M. W. Wu and M. Kuwata-Gonokami, Solid State Commun. 121, 509 (2002).

7 O. Z. Karimov, G. H. John, R. T. Harley, W. H. Lau, M. E. Flatté, M. Henini, and R. Airey, Phys. Rev. Lett. 91, 246601 (2003).

8 S. Döhrmann, D. Hägele, J. Rudolph, M. Bichler, D. Schuh, and M. Oestreich, Phys. Rev. Lett. 93, 147405 (2004).

9 D. Hägele, S. Döhrmann, J. Rudolph, and M. Oestreich, Adv. Solid State Phys. 45, 253 (2005).

10 O. D. D. Couto, Jr., F. Iikawa, J. Rudolph, R. Hey, and P. V. Santos, Phys. Rev. Lett. 98, 036603 (2007).

11 O. D. D. Couto, Jr., R. Hey, and P. V. Santos, Phys. Rev. B 78, 153305 (2008).

12 P. S. Eldridge, W. J. H. Leyland, P. G. Lagoudakis, O. Z. Karimov, M. Henini, D. Taylor, R. T. Phillips, and R. T. Harley, Phys. Rev. B 77, 125344 (2008).

13 V. V. Bel'kov, P. Olbrich, S. A. Tarasenko, D. Schuh, W. Wegscheider, T. Korn, C. Schüller, D. Weiss, W. Prettl, and S. D. Ganichev, Phys. Rev. Lett. 100, 176806 (2008).

14 P. Olbrich, J. Allerdings, V. V. Bel'kov, S. A. Tarasenko, D. Schuh, W. Wegscheider, T. Korn, C. Schüller, D. Weiss, and S. D. Ganichev, Phys. Rev. B 79, 245329 (2009).

15 G. M. Müller, M. Römer, D. Schuh, W. Wegscheider, J. Hübner, and M. Oestreich, Phys. Rev. Lett. 101, 206601 (2008).

16 Y. Zhou and M. W. Wu, Solid State Commun. 149, 2078 (2009).

17 S. A. Tarasenko, Phys. Rev. B 80, 165317 (2009).

18 X. Cartoixà, D. Z.-Y. Ting, and Y.-C. Chang, Phys. Rev. B 71, 045313 (2005).

19 M. I. D'yakonov and V. I. Perel', Zh. Eksp. Teor. Fiz. 60, 1954 (1971) [Sov. Phys. JETP 33, 1053 (1971)]; Fiz. Tverd. Tela (Leningrad) 13, 3581 (1971) [Sov. Phys. Solid State
13, $3023(1972)]$.

20 M. W. Wu and H. Metiu, Phys. Rev. B 61, 2945 (2000); for review: M. W. Wu, J. H. Jiang, and M. Q. Weng, arXiv:1001.0606 and references therein.

21 G. Dresselhaus, Phys. Rev. 100, 580 (1955).

22 Y. A. Bychkov and E. I. Rashba, J. Phys. C 17, 6039 (1984); JETP Lett. 39, 78 (1984).

23 R. Winkler, Phys. Rev. B 69, 045317 (2004).

24 G. L. Bir, A. G. Aronv, and G. E. Pikus, Zh. Eksp. Teor. Fiz. 69, 1382 (1975) [Sov. Phys. JETP 42, 705 (1976)].

25 E. Y. Sherman, Appl. Phys. Lett. 82, 209 (2003); Phys. Rev. B 67, 161303(R) (2003).

26 M. M. Glazov and E. Y. Sherman, Phys. Rev. B 71, 241312(R) (2005).

27 M. Q. Weng and M. W. Wu, Phys. Rev. B 70, 195318 (2004).

28 J. Zhou, J. L. Cheng, and M. W. Wu, Phys. Rev. B 75, 045305 (2007).

29 D. Stich, J. H. Jiang, T. Korn, R. Schulz, D. Schuh, W. Wegscheider, M. W. Wu, and C. Schüller, Phys. Rev. B 76, 073309 (2007).

30 M. M. Glazov and E. L. Ivchenko, Pis'ma Zh. Eksp. Teor. Fiz. 75, 476 (2002) [JETP Lett. 75, 403 (2002)]; Zh. Eksp. Teor. Fiz. 126, 1465 (2004) [JETP 99, 1279 (2004)].

31 W. J. H. Leyland, G. H. John, R. T. Harley, M. M. Glazov, E. L. Ivchenko, D. A. Ritchie, I. Farrer, A. J. Shields, and M. Henini, Phys. Rev. B 75, 165309 (2007).

${ }^{32}$ X. Z. Ruan, H. H. Luo, Y. Ji, Z. Y. Xu, and V. Umansky, Phys. Rev. B 77, 193307 (2008).

33 L. H. Teng, P. Zhang, T. S. Lai, and M. W. Wu, Europhys. Lett. 84, 27006 (2008).

34 E. T. Yu, J. O. McCaldin, and T. C. McGill, Solid State Phys. 46, 1 (1992).

${ }^{35}$ H. Haug and A. -P. Jauho, Quantum kinetics in Transport and Optics of Semiconductors (Springer, Berlin, 1998).

${ }^{36}$ Whether the distribution of $\alpha_{\mathrm{R}}$ is chosen to be the Gaussian or Lorentzian distribution function does not make any difference to our calculation.

37 From Ref. 15, it is known that the QWs are modulation doped in the middle of barrier layer and the width of barrier layer is $80 \mathrm{~nm}$. Since the interaction between electron and impurity in the adjacent doping layer is stronger, the effective distance can be estimated roughly to be 20-40 $\mathrm{nm}$. Thus the fitting value of $z_{0}$ we obtain is in the reasonable range.

38 Due to the different definitions of $\gamma_{\mathrm{D}}$, the value of $\gamma_{\mathrm{D}}$ given here is half of that reported in Ref. 28 .

39 A. N. Chantis, M. van Schilfgaarde, and T. Kotani, Phys. Rev. Lett. 96, 086405 (2006).

40 For lists of $\gamma_{\mathrm{D}}$ calculated and measured via various methods, see Ref. 39 and the supplementary information of [J. J. Krich and B. I. Halperin, Phys. Rev. Lett. 98, 226802 (2007)].

41 I. L. Aleǐner and E. L. Ivchenko, JETP Lett. 55692 , 
(1992); E. L. Ivchenko, A. Y. Kaminski, and U. Rössler, Phys. Rev. B 545852 (1996); T. Guettler, A. L. C. Triques, L. Vervoort, R. Ferreira, P. Roussignol, P. Voisin, D. Rondi, and J. C. Harmand, Phys. Rev. B 58 R10179 (1998).

42 This criterion is always satisfied for the cases in Fig. 1

${ }^{43}$ It is noted that $\Delta_{\mathrm{R}}$ also can be influenced by temperature. However, since the variation of $\Delta_{R}$ with temperature is slower than that of the scattering and inhomogeneous broadening in most situation, our calculation with the fixed $\Delta_{\mathrm{R}}$ is adequate.

44 G. F. Giulianni and G. Vignale, Quantum Theory of the Electron Liquid (Cambridge University Press, Cambridge, England, 2005). 